\title{
Lipid peroxides induce early onset of catagen phase in murine hair cycles
}

\author{
ATSUSHI NAITO, TATSUYUKI MIDORIKAWA, TERUHIKO YOSHINO and MOTOYASU OHDERA
}

\author{
Biological Science Research Laboratories, Research and \\ Development Headquarters, LION Corporation, Odawara, Japan
}

Received April 7, 2008; Accepted June 19, 2008

DOI: 10.3892/ijmm_00000078

\begin{abstract}
The precise mechanisms of alopecia, a pathophysiological disorder with negative psychological implications, are unknown. Androgen and hereditary predisposition are major causes, but the condition is also affected by stress, an irregular diet and high levels of sebum secretion. We focused on oxidative stress and analyzed the effect of the lipid peroxides on hair follicles. Our first observation was that the topical application of linolein hydroperoxides, one of the lipid peroxides, lead to the early onset of the catagen phase in murine hair cycles. Furthermore, by using TUNEL staining we found that lipid peroxides induced apoptosis of hair follicle cells. They also induced apoptosis in human epidermal keratinocytes by up-regulating apoptosis-related genes. These results indicated that lipid peroxides, which can cause free radicals, induce the apoptosis of hair follicle cells, and this is followed by early onset of the catagen phase. These observations may provide insight into the mechanisms underlying the development of alopecia in humans.
\end{abstract}

\section{Introduction}

Hair follicles are miniature hair-shaft-producing organs that have regular cycles of regeneration known as hair cycles. These phases are growth (anagen), regression (catagen) and rest (telogen) (1-3). Hair follicles are composed primarily of epithelial and dermal components, and the hair cycle is a coordinated and complex process, dependent on the interaction of these components $(4,5)$.

Hair loss is the result of premature entry into the catagen phase and the main causes of hair loss are the presence of androgen and hereditary predisposition (6). It has long been suggested that stress, irregular diet and sebum lipids are also involved in the progression of baldness.

Sebum lipids are secreted from sebaceous glands and hormonal changes occurring in the body after puberty cause

Correspondence to: Dr Atsushi Naito, Biological Science Research Laboratories, Research and Development Headquarters, LION Corporation, 100 Tajima, Odawara, Kanagawa 256-0811, Japan

E-mail: a-naito@lion.co.jp

Key words: lipid peroxides, alopecia, hair cycle, apoptosis these glands to become hyperactive (7). Secreted sebum lipids play physical and chemical roles in protecting and moisturizing the hair and skin, but unsaturated fatty acids in sebum lipids are easily converted to lipid peroxides when exposed to oxygen and ultraviolet irradiation (8). Lipid peroxidation has received much attention in connection with its pathological effects and contributions to aging and diseases such as atherosclerosis and cancer (9-17). There are several reports on the effect of lipid peroxides on skin. Saint-Leger et al, for example, showed that squalene oxide may play an important part in the pathology of comedones (18). Chiba et al demonstrated that repetitive topical application of squalene hydroperoxides increased skin roughness and wrinkle formation in hairless mice (19).

Although the harmful effects of lipid peroxides on skin have been well investigated, little is known about the effect of lipid peroxides on hair growth. There is experimental evidence that squalene, a natural component of human sebum, causes reversible hair loss in rabbits and guinea pigs (20), but some of this evidence is not quantitative. In the present study we focused on the lipid peroxides that are produced when sebum lipid on the skin of the scalp is exposed to ultraviolet light (8). To investigate the relationship between these lipid peroxides and hair loss, we studied the effect of linolein hydroperoxides on the hair cycles of mice.

\section{Materials and methods}

Chemicals and animals. Linolein hydroperoxides purchased from Cayman Chemical Co. (MI, USA) were used as the model of lipid peroxides, and 8-week-old C57BL/6 mice were purchased from Japan SLC (Shizuoka, Japan). They were maintained in an air-conditioned room with light from 9 a.m. to 9 p.m. The room temperature, $\left(22 \pm 1^{\circ} \mathrm{C}\right)$ and humidity, $(\sim 60 \%)$ were controlled automatically. Laboratory pellet chows (CE2; CLEA Japan Inc., Tokyo, Japan) and water were freely available.

Cell preparation. For TUNEL staining and gene expression analysis, human epidermal keratinocytes $\left(6 \times 10^{5}\right.$ cells $)$ (Kurabo Industries Ltd., Osaka, Japan) were seeded in dishes $60 \mathrm{~mm}$ in diameter (Sumitomo Bakelite Co. Ltd., Akita, Japan) and were cultured in Humedia KG-2 (Kurabo Industries Ltd.). After reaching subconfluency, these cells were treated with $1 \mu 1$ of linolein hydroperoxides [0.1\% (w/v)] for $24 \mathrm{~h}$. 


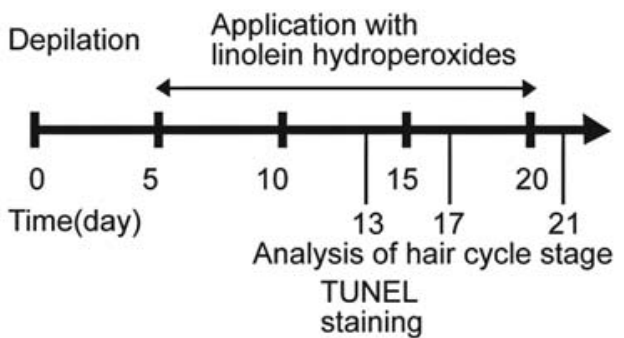

Figure 1. Protocol of topical application of linolein hydroperoxides. Eightweek-old C57BL/6 mice were depilated (day 0). Linolein hydroperoxides $[0.1 \%(\mathrm{w} / \mathrm{v})](0.1 \mathrm{ml}$ per day) were applied to dorsal skin from days 5 to 20 after depilation. The skins were harvested on days 13,17 and 21 after depilation. Ethanol was applied as a control.

Tissue sample preparation. The anagen phase was induced by using, with minor modifications of the method previously described (21), depilatory wax (Anschone; Imyu Co., Tokyo, Japan) to remove the dorsal hair of 8 -week-old C57BL/6 mice.

Skin tissues including samples of the epidermis, dermis and hair follicles were harvested on specific days (Fig. 1). In order to evaluate the effect of linolein hydroperoxides on the onset of the catagen phase, we applied $0.1 \mathrm{ml}$ per day of an ethanolic solution of linolein hydroperoxides $[0.1 \%(\mathrm{w} / \mathrm{v})]$ topically to the dorsal skin from days 5 to 20 after depilation. The samples assessed to evaluate the hair cycle stages were harvested on days $13(n=4), 17(n=4)$ and $21(n=4)$ after depilation. Samples harvested on day 13 were also used for detection of apoptosis. The skins of the control mice were treated with $0.1 \mathrm{ml}$ per day of ethanol.

Histology and histomorphometry of hair follicles. Dorsal skin from the neck region was harvested perpendicular to the paravertebral line to obtain longitudinal hair follicle sections. The samples were embedded in paraffin. Paraffin sections were routinely stained with hematoxylin and eosin or were TUNEL-stained for the detection of apoptosis. The hair cycle stage of each hair follicle was assessed using morphological criteria (22). A minimum of 50 hair follicles per mouse were assessed.

\section{TUNEL staining}

Tissue samples: The TUNEL reaction was performed using the Apoptag ${ }^{\circledR}$ Fluorescein In Situ Apoptosis Detection Kit (Chemicon, Temecula, CA, USA) as described by the manufacturer. Tissue sections were deparaffinized and treated with

A

control

Day 13

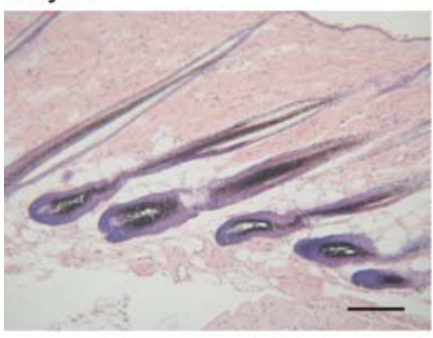

linolein hydroperoxides

Day 13

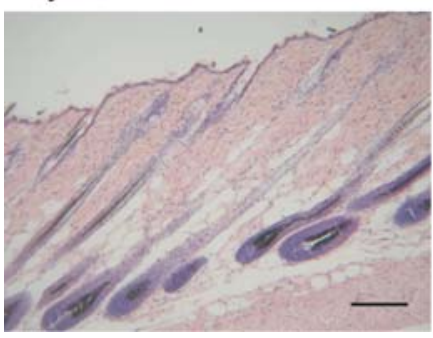

Day 17

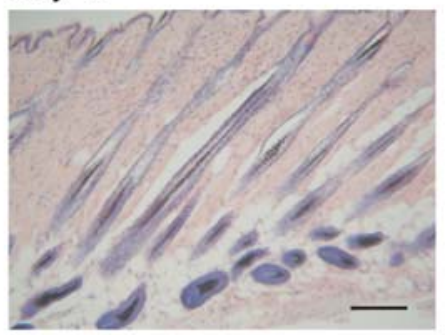

Day 17

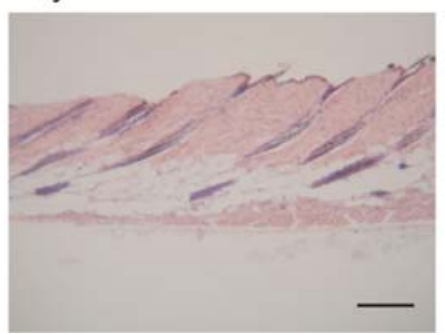

Day 21

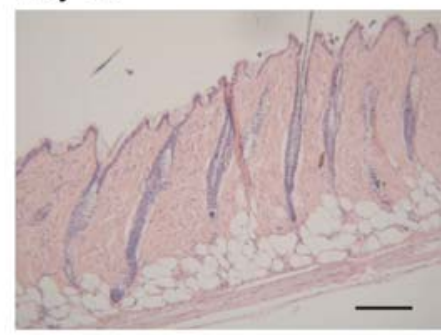

Day 21

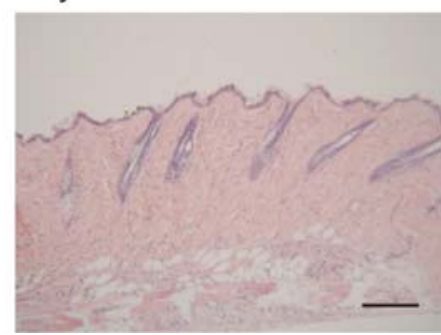

B

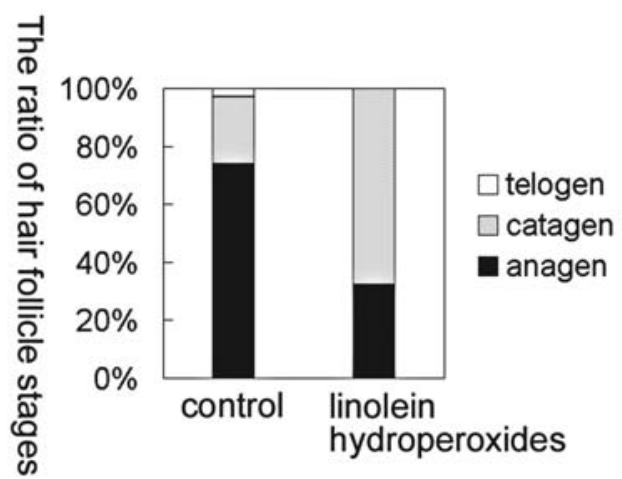

Figure 2. Effect of linolein hydroperoxides on the mouse hair cycle. (A) Representative examples of histochemical features of the hair follicles of mice treated with $0.1 \%$ linolein hydroperoxides $(0.1 \mathrm{ml}$ per day) and control mice (treated with ethanol), 13, 17 and 21 days after depilation $(n=4$; scale bar, $100 \mu \mathrm{m}$ ). Hair follicle features differ between the two groups on days 17 and 21 . (B) The ratio of hair follicle stages at day 17 after depilation. A minimum of 50 hair follicles per mouse were counted, and their hair cycle stages assessed based on the classification of Müller-Röver et al (22) (anagen, anagen I-VI; catagen, catagen I-VIII; and telogen). 


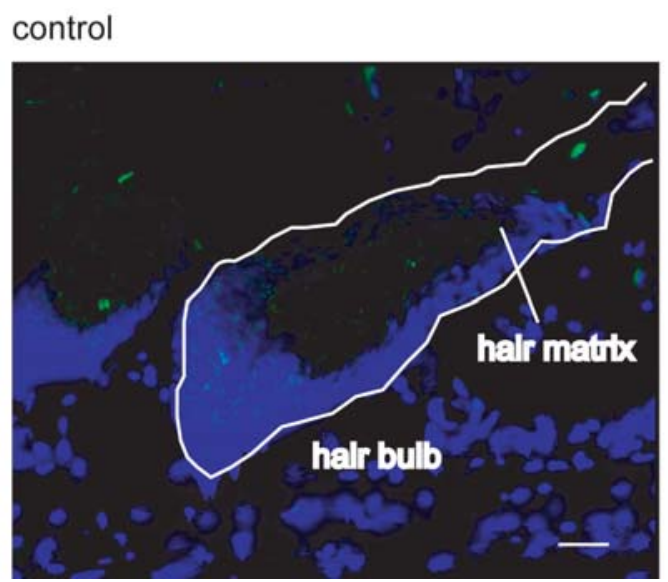

linolein hydroperoxides

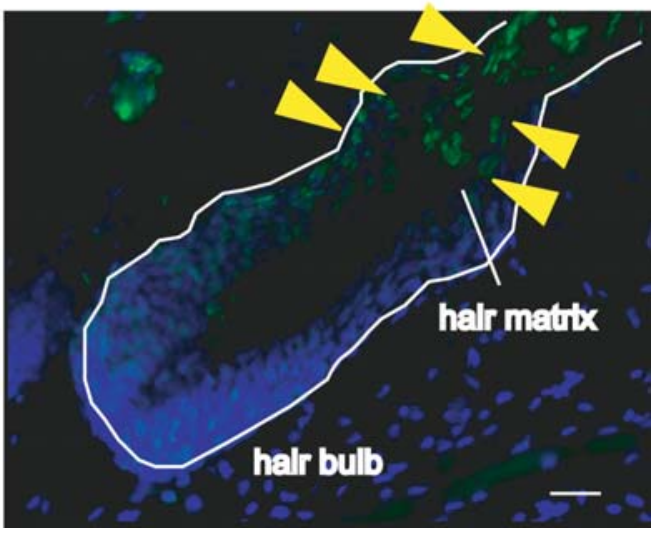

Figure 3. Detection of apoptosis induced in hair follicles 13 days after depilation. Apoptotic hair follicle cells were detected with TUNEL staining (green fluorescence). The hair follicles of mice treated with linolein hydroperoxides had more apoptotic cells in the hair matrix than those of control mice (shown by arrowheads). DAPI was used for counterstaining (blue fluorescence). Scale bar, $10 \mu \mathrm{m}$.

$20 \mu \mathrm{g}$ proteinase $\mathrm{K}$ per $\mathrm{ml}$ for $15 \mathrm{~min}$ at $37^{\circ} \mathrm{C}$. After rinsing with phosphate-buffered saline, sections were incubated for $10 \mathrm{sec}$ at room temperature in an equilibration buffer and then incubated at $37^{\circ} \mathrm{C}$ for $1 \mathrm{~h}$ in a reaction buffer containing TdT enzyme. The reaction was terminated by transferring the slides to a stop/wash buffer. After washing, sections were covered with anti-digoxigenin-fluorescein and incubated for $30 \mathrm{~min}$ at room temperature, avoiding exposure to light. Sections were then counterstained with 4',6-diamino-2phenylindole dihydrochloride (DAPI).

Cell samples: After keratinocytes had been fixed in ethanol for $10 \mathrm{~min}$, the TUNEL reaction was performed using the Apoptag Fluorescein In Situ Apoptosis Detection Kit.

Real-time quantitative RT-PCR. Total RNA was obtained from cultured keratinocytes by using an RNeasy Mini Kit (Qiagen, Valencia, CA, USA). To remove any possible DNA contamination, DNA was digested with a TURBO DNAfree $^{\mathrm{TM}}$ Kit (Ambion, Austin, TX, USA). We purified high quality total RNA (A260/A230 ratios were >1.8). A $1.5 \mu \mathrm{g}$ sample of total RNA was subjected to reverse transcription using MMLV reverse transcriptase RNaseH Minus (Toyobo Co. Ltd., Osaka, Japan) and primed with a random primer (Toyobo Co. Ltd.). Real-time quantitative RT-PCR was performed using a SYBR ${ }^{\circledR}$-Green PCR Master Mix (Toyobo Co. Ltd.) and fluorescence was measured with a GeneAmp 5700 detection system (Applied Biosystems, Foster City, CA, USA). The PCR primers used in this study were apaf1: 5'-CCAAGAAGCTGAGCGAGTGTCT-3' and 5'-CCAGTT GAAGTTGCCGTCAGA-3'; Bax: 5'-CCAAGAAGCTGA GCGAGTGTCT-3' and 5'-CCAGTTGAAGTTGCCGTC AGA-3'; Bcl2: 5'-CCCTCCAGATAGCTCATTTCAT TAAG-3' and 5'-TGCAATCCACTGTCACTCTTGC-3'; caspase 8: 5'-CTGATTCAGAGGAGCAACCCTATTTA-3' and 5'-GCAAAGTGACTGGATGTACCAGGT-3'; caspase 9: 5'-ACTCTACTTTCCCAGGTTTTGTTTCC-3' and 5'-CCC TTTCACCGAAACAGCATT-3'; caspase 10: 5'-GCCCTA GACTGGCTGAAAAACC-3' and 5'-AAGTCAGCCT CGGCAGGAA-3'; p53: 5'-TGCAATAGGTGTGCGTC AGA-3' and 5'-CTCCCAAACATCCCTCACAGTA-3';
GAPDH: 5'-TTGTCAAGCTCATTTCCTGGTATG-3' and 5'-TCCACCACCCTGTTGCTGTA-3'. Up to 40 cycles of amplification were performed, with each cycle consisting of $94^{\circ} \mathrm{C}$ for $15 \mathrm{sec}, 60^{\circ} \mathrm{C}$ for $15 \mathrm{sec}$, and $72^{\circ} \mathrm{C}$ for $30 \mathrm{sec}$. Each gene expression level was compared using GAPDH gene expression as an internal control. Data were pooled from three independent experiments, and the statistical significance of differences was determined by using a two-sided Student's ttest. $\mathrm{P}<0.05$ was considered significant.

\section{Results}

Lipid peroxides lead to the early onset of catagen phase. The hair cycle changes and differences between the control and treated mice are shown in Fig. 2A. Although no differences between the histochemical features of the hair follicles of the two groups were observed on day 13 after depilation, degenerated hair follicles were found in the linolein hydroperoxidetreated mice on day 17. On day 21 after depilation, the hair follicles of the control mice were still in the catagen phase, whereas those of the treated mice were in the telogen phase.

To determine the quantity of hair follicles formed, we evaluated the proportions of hair follicles in the anagen, catagen and telogen phases in each tissue sample on day 17 after depilation. The results are shown in Fig. 2B. Approximately $70 \%$ of the hair follicles from the control mice were still in the anagen phase, whereas $70 \%$ of those from the linolein hydroperoxide-treated mice had already entered the catagen phase. The proportion of hair follicles in the catagen phase was higher for treated than it was for control mice. Topically applied linolein hydroperoxides thus led to early onset of the catagen phase in murine hair cycles.

Lipid peroxides induce apoptosis in murine hair follicles. On day 13 after depilation there were few apoptotic cells in the hair follicles of the control mice but these cells (green fluorescence) were detected in the hair matrix and epithelial cells in the hair follicles of the treated mice (Fig. 3). Linolein hydroperoxides thus induced apoptosis in the hair matrix cells before the onset of the catagen phase. 
control

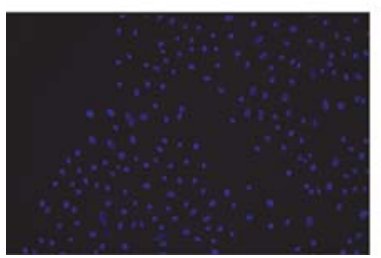

Figure 4. Detection of apoptosis in cultured keratinocytes. Apoptotic keratinocytes were detected with TUNEL staining (green fluorescence). No apoptotic cells were detected in control keratinocytes. Linolein hydroperoxides induced apoptosis in cultured keratinocytes. DAPI was used for counterstaining (blue fluorescence).

Lipid peroxides induce apoptosis in keratinocytes. Apoptosis was observed in the keratinocytes and hair follicle cells of mice treated with linolein hydroperoxides but apoptotic cells were not detected in control keratinocytes (Fig. 4)

Lipid peroxides regulate the expression of apoptosis-related genes in cultured keratinocytes. As linolein hydroperoxides induced apoptosis in the hair matrix and epithelial cells of the hair follicles, we examined the expression of apoptosisrelated genes in the epithelial cells. As shown in Fig. 5, the expression level of $B c l 2$ ( 0.29 fold), which inhibits apoptosis, was down-regulated in the keratinocytes treated with linolein hydroperoxides, and the expression levels of apaf1 (1.53 fold), Bax (2.07 fold), p53 (3.45 fold), caspase 8 (1.93 fold), caspase 9 (2.28 fold) and caspase 10 (3.98 fold) in those cells were up-regulated. Thus linolein hydroperoxides, which induced apoptosis in the keratinocytes, also caused the expression of these apoptosis-related genes to be up-regulated.

\section{Discussion}

In this study we investigated the relationship between the hair cycle and lipid peroxides formed from lipids secreted by sebaceous glands. Human sebaceous glands secrete a lipid mixture containing squalene, wax esters, cholesterol esters,

triglycerides and possibly some free cholesterol (23). Triglycerides produce free fatty acids by catalytic reactions occurring in the presence of bacterial hydrolases (24). The composition of free fatty acids on the surface of the skin and scalp has been extensively studied by Kotani and Kusu (25). Myristic, palmitic, oleic, stearic and linoleic acid are distributed at relatively high concentrations on the scalp. In this study we used linolein hydroperoxides as a model for lipid peroxides. Linolein hydroperoxides are a mixture of 132 possible isomers of mono-, di- and tri-hydroperoxides produced from the autoxidation of trilinolein. Each day $0.1 \mathrm{mg} /$ day $\left(10 \mu \mathrm{g} / \mathrm{cm}^{2}\right)$ of linolein hydroperoxides were applied to the back skin of the mice $(2.5 \times 4 \mathrm{~cm})$. Although the sebum level on the human scalp depends on the time after a shampoo, the average level has been reported to be $\sim 200 \mu \mathrm{g} / \mathrm{cm}^{2}$ (26). In addition, Kohno reported that sunlight oxidizes $\sim 1 \%$ of the total squalene on the human scalp (27). Even though the oxidation rate differs depending on the variety of constituents of sebum, the amount of linolein hydroperoxides applied to the murine back skin each day in this study was $\sim 5$ times the amount of oxidized sebum usually found on the human scalp.

Our finding that linolein hydroperoxides induce the catagen phase in the murine hair cycle suggests that lipid peroxides cause hair loss. The excretion of sebum, which is the source of lipid peroxides, is stimulated by androgen. Increased levels of hydroperoxides as well as increased hair loss are experienced during senescence (28). It is therefore possible that androgen acts as a trigger for hair loss. The primary target cells of androgen in the hair follicle are the dermal papilla cells, which mediate the signals to the follicular epithelial cells (29). In this study, the apoptotic cells of mice treated with linolein hydroperoxides were found not in the dermal papilla cells but in the hair matrix and epithelial cells in the hair follicles. This suggests that linolein hydroperoxides affected the hair matrix and epithelial cells directly through hair shafts and induced apoptosis. Lipid hydroperoxides also induced apoptosis in human vesical fistula, human microvascular and mouse myogenic $\mathrm{C} 2 \mathrm{C} 12$ cells $(30,31)$. The lipid hydroperoxides have a common effect on these and on hair follicle cells.

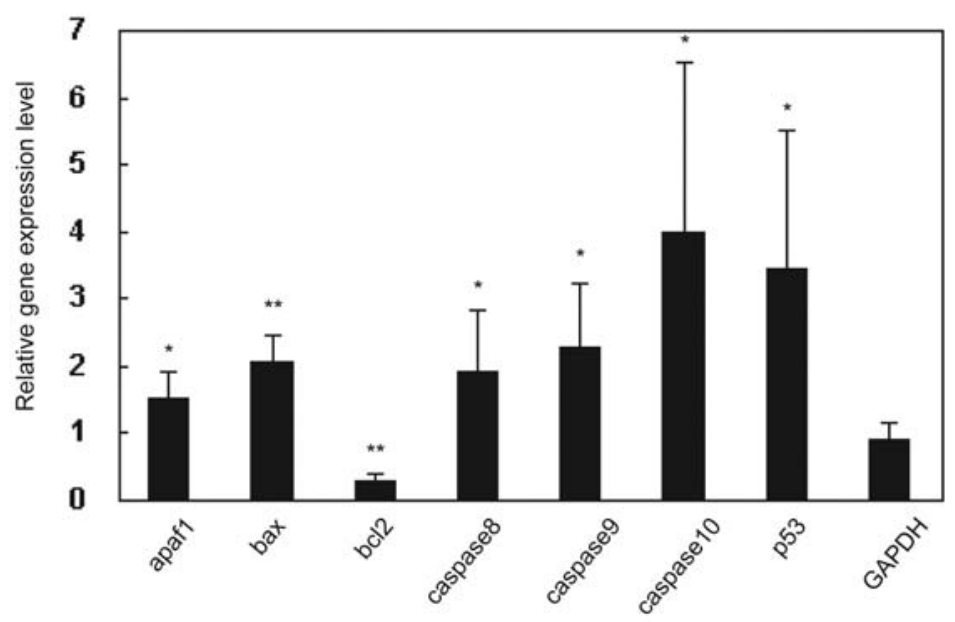

Figure 5. Expression of apoptosis-related genes in keratinocytes ( $\mathrm{n}=3$ ). Changes in the expression of apaf1, Bax, Bcl2, caspase 8, caspase 9, caspase 10 and p53 in control and treatment-group keratinocytes were evaluated by using quantitative RT-PCR analysis. Each gene expression level was compared using GAPDH gene expression as an internal control. Data were pooled from three independent experiments, and the statistical significance of differences was determined by using Student's t-test. $\mathrm{P}<0.05$ was considered statistically significant: ${ }^{* *} \mathrm{P}<0.01$ and ${ }^{*} \mathrm{P}<0.05 ;$ mean $\pm \mathrm{SD}$. 
Since the culture conditions of hair matrix and epithelial cells are not established, we previously investigated the effect of linolein hydroperoxides on the expression of apoptosisrelated genes by using human keratinocytes. The expression level of $p 53$, known as the regulator of apoptosis, was upregulated. In addition, the expression levels of all the genes which we measured, including those of $B c l 2$ and the caspases, were changed in ways that induce apoptosis. Considering these results and the reported retardation of hair follicle regression in the $p 53$ knockout mouse (32), it can be inferred that p53 is an important factor involved in the control of hair cycles.

Lipid hydroperoxides produce reactive oxygen species (ROS), such as peroxyl and alkoxy radicals, during chain reactions. It was suggested that ROS induce the apoptosis of hair matrix and epithelial cells by stimulating the expression of p53. Cells generally have an antioxidant system with oxidative-stress-response genes, such as those for glutathione S-transferase and quinine oxidoreductase, whose expression has been reported to be regulated by NF-E2-related factor 2 transcriptional factor (Nrf2) (33). As p53 suppresses the Nrf2-mediated transcription of antioxidant response genes (34), it was suggested that ROS are not decreased in hair follicles. As a result, disruption of the balance of biological defence mechanisms leads to apoptosis in the hair follicles.

Since we used linolein hydroperoxides as a model of lipid peroxides and the sebum composition differs between mouse and man, it is necessary to investigate the effect of oxidative products of human sebum on the human hair follicles. Nonetheless, we found that linolein hydroperoxides induced the catagen phase in murine hair cycles, which indicates that lipid peroxidation may play a role in the pathogenesis of hair loss.

\section{Acknowledgements}

We thank Prof. Akiyoshi Fukamizu (Center for Tsukuba Advanced Research Alliance, Graduate School of Life and Environmental Sciences, University of Tsukuba) for helpful discussion and also thank Fukamizu laboratory members for encouraging support and advice.

\section{References}

1. Chase HB: Growth of the hair. Physiol Rev 34: 113-126, 1954.

2. Paus R: Control of the hair cycle and hair diseases as cycling disorders. Curr Opin Dermatol 3: 248-258, 1996.

3. Stenn KS and Paus R: Controls of hair follicle cycling. Physiol Rev 81: 449-494, 2001.

4. Couchman JR and Gibson WT: Expression of basement membrane components through morphological changes in the hair growth cycle. Dev Biol 108: 290-298, 1985.

5. Couchman JR, King JL and McCarthy KJ: Distribution of two basement membrane proteoglycans through hair follicle development and the hair growth cycle in the rat. J Invest Dermatol 94: 65-70, 1990.

6. Hamilton JB: Male hormone stimulation is a prerequisite and an incitant in common baldness. Am J Anat 71: 451-480, 1942.

7. Cotterill JA, Cunliffe WJ, Williamson B and Bulusu L: Age and sex variation in skin surface lipid composition and sebum excretion rate. Br J Dermatol 87: 333-340, 1972.

8. Mudiyanselage SE, Hamburger M, Elsner P and Thiele JJ: Ultraviolet a induces generation of squalene monohydroperoxide isomers in human sebum and skin surface lipid in vitro and in vivo. J Invest Dermatol 120: 915-922, 2003.

9. Pryor WA: The formation of free radical and the consequences of their reactions in vivo. J Photochem Photobiol 28: 787-801, 1978.
10. Yagi K: Lipid peroxides and human diseases. Chem Phys Lipids 45: 337-351, 1987.

11. Cerutti PA: Prooxidant states and tumor promotion. Science 227: 375-381, 1985.

12. Praper HH and Bird RP: Antioxidants and cancer. J Agric Food Chem 32: 433-435, 1984

13. Leibovitez BE and Siegel BV: Aspect of free radical reaction in biological systems. Aging J Gerontol 35: 45-56, 1980.

14. Harman D, Heidric ML and Eddy DE: Free radical theory of aging: Effect of free-radical-reaction inhibitories on immune response. J Am Geriatr Soc 25: 400-407, 1997.

15. Suzuki T, Miyazawa T, Fujimoto K, Ohtuka M and Tsutsumi M: Age-related accumulation of phosphatidylcholine hydroperoxide in cultured human diploid cells and its prevention by $\alpha$-tocopherol. Lipids 28: 775-778, 1993.

16. Miyazawa T, Suzuki T and Fujimoto K: Age-dependent accumulation of phosphatidylcholine hydroperoxide in the brain and liver of the rat. Lipids 28: 789-793, 1993.

17. Yoshida LS, Miyazawa T, Hatayama I, Sato K, Fujimoto K and Kaneda T: Phosphatidylcholine peroxidation and liver cancer in mice fed a choline-deficient diet with ethionine. Free Radic Biol Med 14: 191-199, 1993

18. Saint-Leger D, Baque A, Lefebvre E, Cohen E and Chivot M: A possible role for squalene in the pathogenesis of acne. In vivo study of squalene oxides in skin surface and intra-comedonal lipids of acne patients. Br J Dermatol 114: 543-552, 1986.

19. Chiba K, Sone T, Kawakami K and Onoue M: Skin roughness and wrinkle formation induced by repeated application of squalene-monohydroperoxide to the hairless mouse. Exp Dermatol 8: 471-479, 1999.

20. Flesch P and Goldstone SB: Local depilatory action of unsaturated compounds; the effect of human sebum on hair growth. J Invest Dermatol 18: 267-287, 1952.

21. Matsuda H, Yamazaki M, Naruto S, Asanuma Y and Kubo M: Anti-androgenic and hair growth promoting activities of lygodii spora (spore of Lygodium japonicum) I. Active constituents inhibiting testosterone 5 $\alpha$-reductase. Biol Pharm Bull 25: 622-626, 2002.

22. Müller-Röver S, Handjiski B, van der Veen C, et al: A comprehensive guide for the accurate classification of murine hair follicles in distinct hair cycle stages. J Invest Dermatol 117: 3-15, 2001.

23. Yamamoto A: Biochemistry and function of human sebaceous lipid. Fragrance J 22: 11-17, 1994.

24. Marples RR, Kligman AM, Lantis LR and Downing DT: The role of the aerobic microflora in the genesis of fatty acids in human surface lipids. J Invest Dermatol 55: 173-178, 1970.

25. Kotani A and Kusu F: HPLC with electrochemical detection for determining the distribution of free fatty acids in skin surface lipids from the human face and scalp. Arch Dermatol Res 294: 172-177, 2002.

26. Saint-Leger D and Leveque JL: A comparative study of refatting kinetics on the scalp and forehead. Br J Dermatol 106: 669-675, 1982.

27. Kohno Y: Reactive oxygen species/free radicals in the skin and development of cosmetics in preventing of skin aging. Fragrance J 11: 35-42, 1993.

28. Lippman RD: Rapid in vivo quantification and comparison of hydroperoxides and oxidized collagen in aging mice, rabbits and man. Exp Gerontol 20: 1-5, 1985.

29. Inui S, Fukuzato Y, Nakajima T, Yoshikawa $K$ and Itami S: Androgen-inducible TGF- $ß 1$ from balding dermal papilla cells inhibits epithelial cell growth: a clue to understanding paradoxical effects of androgen on human hair growth. FASEB J 16: 1967-1969, 2002.

30. Kontush A, Chancharme L, Escargueil-Blanc I, et al: Mildly oxidized LDL particle subspecies are distinct in their capacity to induce apoptosis in endothelial cells: role of lipid hydroperoxides. FASEB J 17: 88-90, 2003.

31. Chiou TJ, Chu ST and Tzeng WF: Protection of cells from menadione-induced apoptosis by inhibition of lipid peroxidation. Toxicology 191: 77-88, 2003.

32. Botchkarev VA, Komarova EA, Siebenhaar F, et al: p53 involvement in the control of murine hair follicle regression. Am J Pathol 158: 1913-1919, 2001.

33. Ikeda H, Serria MS, Kakizaki I, et al: Activation of mouse Piclass glutathione S-transferase gene by Nrf2 (NF-E2-related factor 2) and androgen. Biochem J 364: 563-570, 2002.

34. Faraonio R, Vergara P, Di Marzo D, et al: p53 suppresses the Nrf2-dependent transcription of antioxidant response genes. J 